EPJ manuscript No.

(will be inserted by the editor)

\title{
Non-empirical pairing functional
}

\author{
T. Duguet ${ }^{1, a, b}$ and T. Lesinski ${ }^{2, c}$ \\ ${ }^{1}$ National Superconducting Cyclotron Laboratory and Department of Physics and Astronomy, Michi- \\ gan State University, East Lansing, MI 48824, USA \\ 2 Université de Lyon, F-69003 Lyon, France; Institut de Physique Nucléaire de Lyon, CNRS/IN2P3, \\ Université Lyon 1, F-69622 Villeurbanne, France
}

\begin{abstract}
The present contribution reports the first systematic finite-nucleus calculations performed using the Energy Density Functional method and a nonempirical pairing functional derived from low-momentum interactions. As a first step, the effects of Coulomb and the three-body force are omitted while only the bare two-nucleon interaction at lowest order is considered. To cope with the finite-range and non-locality of the bare nuclear interaction, the ${ }^{1} S_{0}$ channel of $V_{\text {low k }}$ is mapped onto a convenient operator form. Neutron-neutron and protonproton pairing correlations generated in finite nuclei by the direct term of the two-nucleon interaction are characterized in a systematic manner. Eventually, such predictions are compared to those obtained from empirical local functionals derived from density-dependent zero range interactions. The characteristics of the latter are analyzed in view of that comparison and a specific modification of their isovector density dependence is suggested to accommodate Coulomb effects and the isovector trend of neutron gaps at the same time.
\end{abstract}

\section{Introduction}

Low-Energy Nuclear Theory is currently going through an unprecedented revival. First, the explicit link between Quantum Chromodynamics and inter-nucleon interactions is being realized through Effective Field Theory (EFT) based on Chiral Perturbation Theory [1. Second, the recent advent of low-momentum nuclear interactions obtained through the application of Renormalization Group (RG) techniques [2 opens up, for the first time, the possibility to understand properties of heavy nuclei from underlying microscopic interactions. This is of crucial importance in view of the challenge posed by exotic nuclei displaying an unusually large ratio of neutrons over protons. Indeed, many traditional features of nuclei close to the valley of stability tend to be significantly modified as one adds more neutrons to the same element. As a matter of fact, the predictive power of current theoretical methods is rather limited as one goes towards experimentally unknown nuclei. It is mandatory to improve such a situation considering that ambitious experimental programs are being developed around the world to synthesize and study medium-mass neutron-rich nuclei.

The nuclear Energy Density Functional (EDF) approach is the microscopic tool of choice to study medium-mass and heavy nuclei in a systematic manner [3]. Such an approach to finite nuclei strongly relies on the concept of symmetry breaking and is formulated as a two-step

a e-mail: duguet@nscl.msu.edu

b Both authors wish to thank K. Bennaceur for his collaboration on this on-going research program. This work was supported by the U.S. National Science Foundation under Grant No. PHY-0456903.

c e-mail: lesinski@ipnl.in2p3.fr 
method: (i) the Single-Reference (SR) formulation that incorporates static collective correlations associated with symmetry-breaking modes (ii) the Multi-Reference (MR) formulation 1 that further includes quantum correlations associated with the fluctuations of the phase and magnitude of the order parameters of broken symmetries [5. The EDF approach has had many qualitative and quantitative successes over the last twenty years [3] despite its empirical construction by analogy to wave-function-based methods, i.e. Symmetry Unrestricted Hartree-Fock and the Generator Coordinate methods. As recently discovered however, the empirical nature of the MR-EDF method leads to serious pathologies that compromise its past and future predictions $[6$. Corrections to such pathologies are currently being designed 7 . The EDF method is also empirical in the sense that the functionals used so far, e.g. Skyrme [8] or Gogny [9] only have a very loose connection to underlying inter-nucleon interactions. As a result, the predictive power of the method away from the regions where the functional is constrained through the reproduction of selected experimental data is limited.

In the present contribution, we limit ourselves to the SR level and focus on static pairing correlations incorporated through the breaking of $U(1)$ symmetry. Correlations associated with the fluctuations of the phase [10 and the magnitude [11|12] of the pairing gap can be further incorporated at the MR leve 2. Nucleonic pairing has a strong influence on all low-energy properties of nuclei. This encompasses masses, separation energies, deformation, individual excitation spectra and collective excitation modes such as rotations or vibrations. The role of pairing correlations is particularly emphasized when going toward the neutron drip-line because of the proximity of the single-particle continuum.

As for the EDF as a whole, pairing functionals that have been used so far are of empirical character. Their ability to reproduce pairing properties of nuclei close to the valley of stability, e.g. the Odd-Even Mass Staggering (OEMS) [16, moment of inertia of rotational nuclei [17. can be considered as satisfactory. However, detailed and systematic characterizations of those observables and of individual excitation spectra in even-even and odd-even nuclei as well as their dependence on the properties of the pairing functional are still missing. In addition, there are hints from the spreading of the predictions that the predictive power of existing empirical functionals as one goes to more neutron rich nuclei and enters the "next major shell" is very limited [18.

Our long-term objective is the construction of non-empirical energy density functionals derived explicitly from inter-nucleon two-body and three-body interactions [19. As already alluded to, the motivation to do so lies in the fact that empirical EDFs leave unexplained all fitted data and, while they can make accurate predictions for systems and properties that are sufficiently similar to those fitted, they can fail badly for systems and properties that differ significantly from those fitted. Thanks to their suggested perturbative nature [20, lowmomentum potentials (" $V_{\text {low k}}$ ") 2] offer the opportunity to construct energy functionals from bare nuclear interactions for the first tim 3 . As a very first step, we focus here on the pairing part of the nuclear EDF.

The paper is organized as follows. Basic elements of the SR-EDF approach are recalled in section 2 In section 3, the construction of the non-empirical pairing functional from lowmomentum interactions is briefly explained. The corresponding results are reported in section 4 for a set of semi-magic nuclei and compared those obtained from traditional empirical pairing functionals. Our conclusions and perspectives are given in section 5 .

\section{Elements of single-reference EDF formalism}

The SR-EDF including $U(1)$ symmetry breaking takes the form of a generalized Hartree-FockBogoliubov (HFB) formalism where the particle-hole and particle-particle parts of the EDF

\footnotetext{
1 The Quasi-particle Random Phase Approximation is a limit case of the MR-EDF approach [4].

${ }^{2}$ Pairing correlations can also be grasped through symmetry conserving approaches [13 14 15].

3 Here, a two-body (three-body) bare interaction is in principle any interaction that fits the two-body (three-body) scattering phase shifts in a low-energy domain that is physically relevant to low-energy nuclear structure and reproduce the deuteron (triton and ${ }^{3} \mathrm{He}$ ) binding energy.
} 
may resum correlations beyond the strict Hartree, Fock and Bogoliubov diagrams calculated in terms of the bare nuclear Hamiltonian. The energy is postulated under the form a functional $\mathcal{E}\left[\rho, \kappa, \kappa^{*}\right]$ of the (symmetry breaking) one-body density $\rho$ and pairing tensor $\kappa$. The latter density matrices are mapped through an auxiliary product state $|\Phi\rangle$,

$$
\rho_{i j} \equiv\left\langle\Phi\left|\hat{a}_{j}^{\dagger} \hat{a}_{i}\right| \Phi\right\rangle, \quad \kappa_{i j} \equiv\left\langle\Phi\left|\hat{a}_{j} \hat{a}_{i}\right| \Phi\right\rangle,
$$

where $\left\{\hat{a}_{i}^{\dagger}\right\}$ is an arbitrary single-particle basis. The minimization of $\mathcal{E}\left[\rho, \kappa, \kappa^{*}\right]$ under particlenumber constraints enforced through the use of a chemical potential $\lambda$ leads to the generalized HFB equations

$$
\left(\begin{array}{cc}
h-\lambda & \Delta \\
-\Delta^{*}-h^{*}+\lambda
\end{array}\right)\left(\begin{array}{l}
\mathcal{U} \\
\mathcal{V}
\end{array}\right)_{\mu}=E_{\mu}\left(\begin{array}{l}
\mathcal{U} \\
\mathcal{V}
\end{array}\right)_{\mu},
$$

where $(\mathcal{U}, \mathcal{V})_{\mu}$ are the upper and lower components of the Bogoliubov quasi-particle eigenstate whereas $E_{\mu}$ denotes the corresponding quasi-particle energy. Once the quasi-particle eigenstates are obtained, $\rho$ and $\kappa$ can be recalculated and the iterative procedure processed until convergence. The single-particle field $h$ and the pairing field $\Delta$ are obtained through functional derivatives

$$
h_{j i} \equiv \frac{\delta \mathcal{E}}{\delta \rho_{i j}}=h_{i j}^{*}, \quad \Delta_{i j} \equiv \frac{\delta \mathcal{E}}{\delta \kappa_{i j}^{*}}=-\Delta_{j i} .
$$

In a functional HFB scheme, the particle-hole and particle-particle channels can only be defined rigourously at the level of the fields $h$ and $\Delta$, i.e. relatively to the density matrix with respect to which the functional derivative is taken. Indeed, and except for the strict bilinear functional obtained from the Hartree, Fock and Bogoliubov diagrams, the $\kappa$ and $\rho$ dependences are entangled in the energy $\mathcal{E}\left[\rho, \kappa, \kappa^{*}\right]$ and one cannot in general split the functional into a particle-hole and a particle-particle part. The common separation of the EDF into a particlehole part on the one hand and a pairing part on the other has only relied on the very simple functional dependence of standard phenomenological functionals. Indeed, the only (bilinear) term depending on $\kappa$, possibly further depending on $\rho$, has usually been characterized as the particle-particle part of the EDF, the rest then defining its particle-hole part.

\section{Non-empirical pairing functional from low-momentum interactions}

\subsection{Constructive many-body framework}

Instead of postulating the form of the nuclear EDF and fitting it to nuclear data, our long-term goal is to construct it explicitly from many-body techniques formulated in terms of inter-nucleon interactions in the vacuum. Starting from traditional two-body hard-core interactions, such an endeavor has always been perceived as unrealistic because of the non-perturbative nature of the many-body problem and quantitatively unpractical because of the Coester line problem 21. The advent of low-momentum interactions [2] and the better understanding of the role played by three-nucleon forces make such a constructive approach promising for the first time, at least in view of constraining the form and parameters of the nuclear EDF. As a matter of fact, a SR many-body perturbation theory (MBPT) formulated in terms of low-momentum interactions becomes a viable approach to building correlations into the functional 20. This is the path we are going to follow. Within such a scheme, the symmetry-breaking auxiliary state $|\Phi\rangle$ entering the SR-EDF formalism is nothing but the vacuum on top of which the perturbation theory is performed.

As we treat pairing correlations through $U(1)$ symmetry breaking, MBPT must be formulated including anomalous propagators (in terms of Feynman 22 or Goldstone 23 diagrams). At lowest order, the irreducible vertex entering the pairing channel is given by the bare nuclear interaction ; i.e. it corresponds to the strict Bogoliubov diagram. Omitting the three-nucleon interaction for now, the corresponding part of the EDF is thus non local, bilinear in $\kappa$ and $\rho$ independent ; i.e. $\mathcal{E}\left[\rho, \kappa, \kappa^{*}\right] \equiv \mathcal{E}[\rho]+\mathcal{E}^{\kappa \kappa}$, where $\mathcal{E}^{\kappa \kappa}$ denotes the bilinear pairing functional. At the next order, the irreducible pairing vertex involves the so-called polarization diagrams [24 25]. 
We limit ourselves in the present work to constructing the pairing part of the EDF from low-momentum interactions and keeping the remaining part empirical, retaining only the lowest order contribution of the (nuclear part of) the two-body interaction in the pairing energy. Of course, the full consistency of the approach will only be attained when the Coulomb and threenucleon forces are considered, when higher orders are incorporated and all terms of the EDF are generated from underlying interactions. It is worth mentioning that the particle-hole part of the EDF influences pairing properties since pair scattering strongly depends on the characteristics of the single-particle field on top of which it develops. The latter developments are indeed envisioned within our long term research program.

Despite the limitations of the first step undertaken in the present work, a significant amount of relevant physics can be addressed already. For example, pairing correlations generated by the direct term of the two-nucleon interaction have only been characterized so far in one finite nucleus, thanks to a very involved calculation 25. The contribution of the direct term of the two-nucleon interaction to the magnitude and isotopic dependence of finite nuclei pairing gaps is however of general interest. Indeed, it is unique to nuclear systems and trapped cold atoms that the direct term of the inter-particle interaction generates superfluidity ; i.e. superconductivity in electronic systems is due to induced interactions generated by the coupling to lattice phonons. By a straight comparison with experimental data, the characterization initiated in this work will eventually suggest what is missing in the pairing functional beyond the direct term of the two-nucleon interaction.

\subsection{Operatorial mapping of $V_{\text {low } \mathrm{k}}$}

Limiting ourselves to the direct term of the bare two-nucleon interaction brings an extra simplification. As known from scattering phase-shifts, only the ${ }^{1} S_{0}$ channel of the nuclear interaction can generate pairing at sub-saturation densities [26. As a result, all other partial waves can be omitted at this point. Of course, this would not be true beyond lowest order and/or if constructing the particle-hole part of the EDF.

In the present work, the pairing functional is generated from $V_{\text {low k }}$ calculated at a resolution cut-off of $\Lambda \approx 2 \mathrm{fm}^{-1}$. Traditionally, $V_{\text {low } \mathrm{k}}$ is generated through $\mathrm{RG}$ flow equations and takes the form of tables of matrix elements in momentum space for each partial wave. For practical reasons, we need an operator representation of the interaction which makes systematic EDF calculations of nuclei tractable. To capture the finite range and non-locality of $V_{\text {lowk }}$ in a way that remains numerically tractable, we produce a rank- $n$ separable representation of the interaction. Thus, focusing on the ${ }^{1} S_{0}$ channel, the spatial part of the interaction is represented as

$$
\left\langle\mathbf{r}_{1} \mathbf{r}_{2}\left|V_{\text {low }}^{S}\right| \mathbf{r}_{3} \mathbf{r}_{4}\right\rangle \equiv \sum_{\alpha, \beta=1}^{n} G_{\alpha}\left(s_{12}\right) \lambda_{\alpha \beta} G_{\beta}\left(s_{34}\right) \delta\left(\mathbf{R}_{12}-\mathbf{R}_{34}\right)
$$

where $\mathbf{R}_{12}=\left(\mathbf{r}_{1}+\mathbf{r}_{2}\right) / 2$ and $\mathbf{s}_{12}=\mathbf{r}_{1}-\mathbf{r}_{2}$ are the center-of-mass and relative coordinates of the interacting-particle pair, respectively. The rank $n$, the form factors $G_{\alpha}(r)$ and the coupling parameters $\lambda_{\alpha \beta}$ have to be specified and optimized. In fact, the nuclear interaction is almost separable in the ${ }^{1} S_{0}$ channel due to the presence of a virtual state at almost zero scattering energy [28. As a result, a rank-1 representation is already quantitatively satisfactory [29] and will be used in the present work. Even though the separable nature of the mapping brings noticeable simplifications [27, the finite-range and non-local vertex cannot be handled easily in any existing HFB code. The high-precision operatorial mapping of $V_{\text {low }}$ and the specificities of our new code 30 will be discussed in detail in a future work 27.

\footnotetext{
4 The low-energy phase shifts generated by the rank-1 separable representation used better match experimental data than is apparent in Fig. 2 of Ref [29. This is because the results shown on that figure were plagued by a numerical error.
} 


\section{First results in semi-magic nuclei}

As already mentioned, the entire EDF is not constructed consistently from low-momentum interactions at this point. Thus, the non-empirical pairing functional is combined to a Skyrme EDF for the particle-hole part; i.e. the SLy5 parametrization 31. The corresponding densitydependent and momentum-independent isoscalar effective mass is equal to $m_{0}^{*}=0.7 \mathrm{~m}$ at saturation density. In section 4.2, the non-empirical pairing functional will be replaced by empirical ones derived from Density Dependent Delta Interactions (DDDI). The calculations are performed with the BSLHFB code [30 in a spherical box of radius $R=20 \mathrm{fm}$ and using a discretized Bessel basis $j_{l}(k r)$ with $k<k_{\max }=4 \mathrm{fm}^{-1}$ and a partial-wave cutoff $j_{\max }=45 / 2$. Using those ingredients, we calculate the properties of about 470 even-even (predicted) spherical nuclei [34].

In the present contribution, we limit ourselves to a single observable related to pairing correlations, i.e. the odd-even mass staggering. In Ref. 27, the analysis will be deepened and several other observables will be discussed. The connection between finite difference mass formulae employed to extract the OEMS and theoretical gaps is less trivial than usually thought 32. In the present case, experimental three-point mass difference formula $\Delta_{q}^{(3)}(N / Z)$ centered on odd $N / Z$, will be compared to straight theoretical gaps calculated in even-even nuclei. While this corresponds to the best zeroth-order comparison, a more advanced treatment [32] will be employed in Ref. 27. to reach precise quantitative conclusions. The actual theoretical measures used are twofold (i) $\Delta_{L C S}^{q}$ is the canonical gap matrix element associated with the lowest canonical quasi-particle energy (ii) $E_{L Q P}^{q}$ is the lowest positive quasi-particle energy solution of Eq. 2 As opposed to $\Delta_{L C S}^{q}, E_{L Q P}^{q}$ also contains a contribution from the underlying single-particle spectrum. Where the two quantities significantly differ corresponds to (sub-)shell closures which must be avoided in the discussion of static pairing correlations.

\subsection{Non-empirical pairing functional}

The upper panel of Fig. 1 displays the comparison between the experimental OEMS and theoretical neutron gaps obtained from the non-empirical functional along isotopic chains of "light" $(\mathrm{Ca}$ and $\mathrm{Ni})$, medium-mass $(\mathrm{Sn})$ and heavy $(\mathrm{Pb})$ elements. One observes that (i) pairing gaps slightly decrease with increasing mass ; (ii) neutron gaps obtained from the non-empirical functional are very close to experimental gaps in the lightest elements (iii) but larger by a few hundreds $\mathrm{keV}$ in tin and lead isotopes ; (iv) theoretical neutron gaps, which cover a wider range of isospin asymmetry than experimental data, display a decreasing slope with $N-Z$ along all isotopic chains.

The agreement between experimental mass differences and the neutron gaps obtained from the non-empirical functional is striking, to a large extent surprising, and to be taken with a grain of salt at this point. Indeed, four crucial elements must be recalled at this point (i) gaps are exponentially sensitive to the interaction strength ; (ii) the non-empirical functional is generated without any adjustment on finite-nucleus data (iii) the non-empirical functional considered at this point is not final since it is only derived at lowest order in the interaction ; i.e. it is not expected to reproduce experimental data, especially in view of the recent claims that spin, isospin and density fluctuations beyond the direct term are expected to impact pairing gaps significantly [25] ; (iv) the present results are based on an empirical particle-hole functional which is not fully consistent with the pairing vertex. We will briefly come back to that below. In any case, and even if the direct term provides neutron gaps of the right order of magnitude in nearly stable nuclei, the three-body force and higher-order effects are likely to be crucial to reach a quantitative agreement with experiment on a nucleus-by-nucleus basis as well as to understand isotopic trends as we go towards more neutron-rich nuclei.

The lower panel of Fig. 1 1displays proton gaps along isotonic chains $N=28,50,82,126$. One observes that experimental proton gaps decrease significantly with increasing mass and are, for a given mass region, smaller than the neutron ones. One also observes that theoretical proton gaps obtained from the (isospin-invariant) non-empirical pairing functional do not significantly 

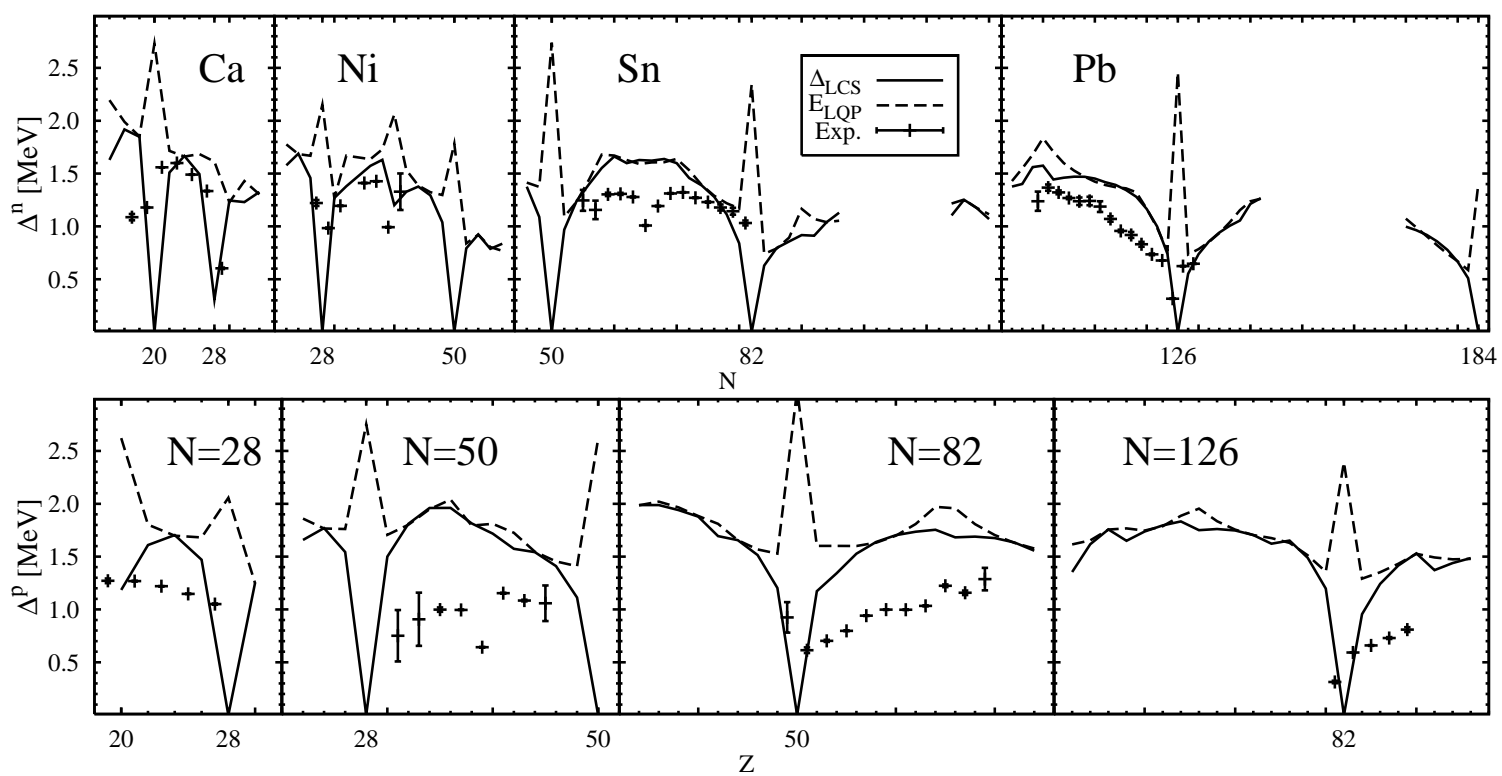

Fig. 1. Experimental three-point odd-even mass difference (crosses) and zeroth-order theoretical pairing gaps obtained from the non-empirical pairing functional (lines). Upper panel: neutron gaps along $\mathrm{Ca}, \mathrm{Ni}, \mathrm{Sn}$ and $\mathrm{Pb}$ isotopic chains. Lower panel: proton gaps along $N=28,50,82$ and 126 isotonic chains. Results are not displayed for nuclei predicted not to be spherical when our criterion 33 is applied to the results of Ref. 34].

decrease with mass, are rather flat as a function on $N-Z$ and significantly overestimate experimental data, a discrepancy which increases with nuclear mass. The difference with respect to the situation previously discussed for neutrons is striking. Qualitatively speaking, the reduction of experimental proton gaps with respect to neutron ones is not visible in the theoretical results. Of course, and even though other missing elements might be invoked, it is likely that the missing Coulomb interaction is mainly responsible for that discrepancy. EDF calculations including Coulomb in the pairing have been extremely rare [35] and it will be of interest to test such a hypothesis 27 .

It is of interest to compare $\Delta_{L C S}^{n}$ obtained in ${ }^{120} \mathrm{Sn}$ to the one reported in Ref. [25, which is the only gap calculated in finite nuclei from a bare two-nucleon interaction prior to the present work. The latter calculation was performed using the SLy4 Skyrme parametrization in the particle-hole channel and the Argonne V18 36 bare interaction in the pairing channel. It happens that the predicted gap was about half of the one obtained here using the separable representation of $V_{\text {low }} 1^{5}$. It is surprising at first considering that both interactions provide identical gaps in infinite nuclear matter when calculated with free single-particle energies [29. The reason for the difference seen in ${ }^{120} \mathrm{Sn}$ relates to the resolution scale (the $\Lambda$ cut-off in $\mathrm{RG}$ terms) at which the two-body interaction employed in the pairing channel and the effective mass characterizing the single-particle field in the particle-hole channel are defined. Such a critical issue will be discussed at length in a forthcoming publication 39. In any case, the relative agreement between experiment and the gaps obtained presently, together with the qualitative discrepancy between the latter gaps and those displayed in Ref. 25], raise the question of the quantitative importance of higher-order effects associated with the coupling to spin, isospin and density fluctuations.

\footnotetext{
${ }^{5}$ We do not quote precise values of the gaps because the calculation of Ref. 25 was done using a reduced spin-orbit coupling strength as opposed to the original SLy4 parametrization. On the other hand, it has been checked that the difference in the spin-orbit coupling is not sufficient to explain the difference in the predicted gaps.
} 

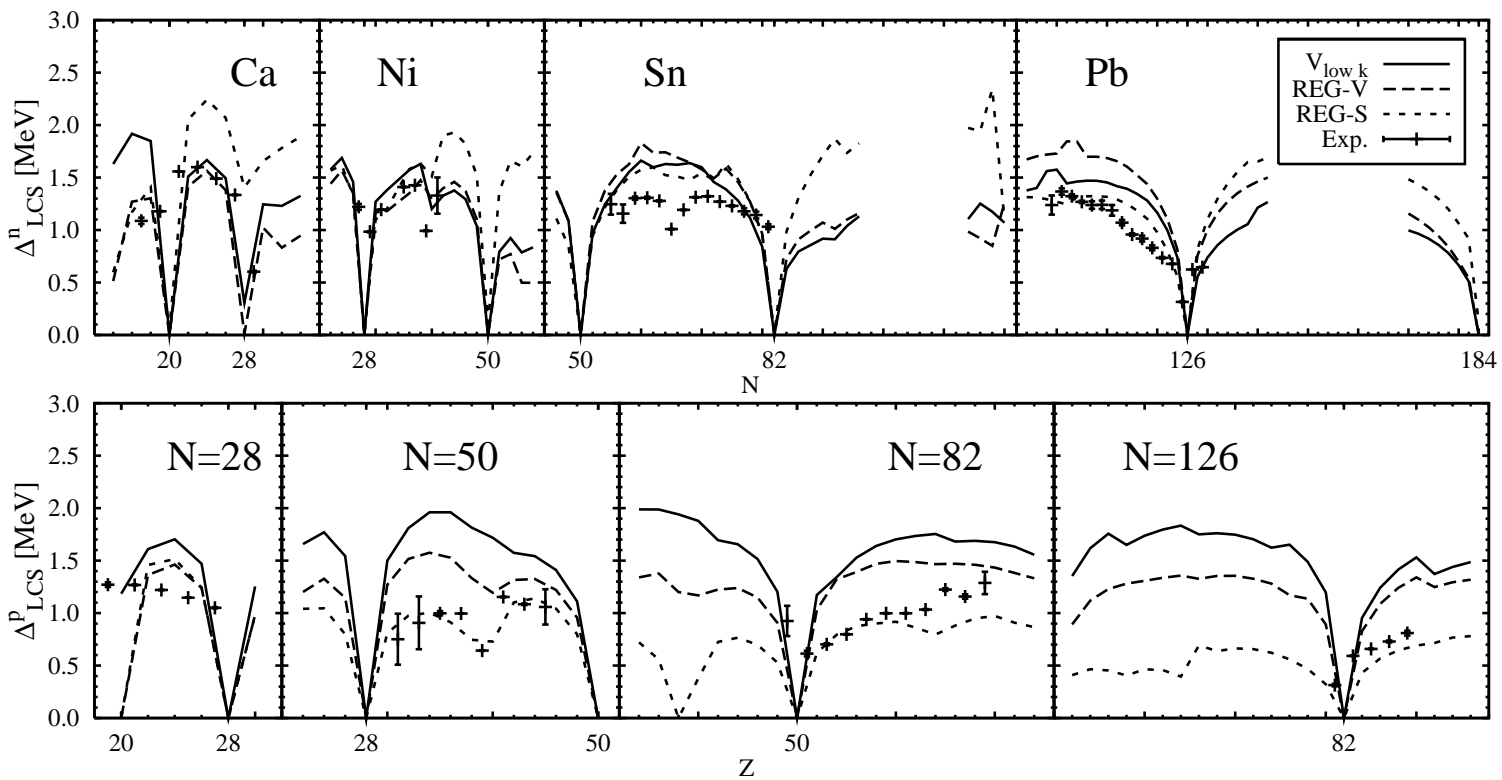

Fig. 2. Same as Fig. 1 for gaps obtained from empirical DDDI pairing functionals (see text).

\subsection{Comparison with empirical pairing functionals}

One of the goals of the present work is to corroborate the successes held by empirical local pairing functionals and to help enriching them in a controlled manner. The latter objective is of particular interest considering that the non-locality of the non-empirical functional makes it numerically demanding and difficult to adapt to 3D codes. By a careful comparison of the results obtained from both approaches, one can thus hope to pin down necessary isoscalar and isovector density dependences as well as genuine gradient corrections ; i.e. explicit finite-range effects beyond the necessary ultra-violet regularization/renormalization of quasi-local pairing functionals. Of course, the "complete" pairing functional will need to resum effects from higherorder contributions and three-body forces and the comparison performed at this point can only account for lowest-order effects from the two-body interaction. In addition, we only focus on the general trends of the OEMS and postpone a detail analysis and the extension to other ground-state observables as well as excited states properties to a future work [27.

For that purpose, we thus consider two empirical functionals derived from DDDI and regularized through a standard cut-off scheme 37. (i) REG-V is a density-independent local functional (ii) REG-S is a local functional depending on the isoscalar density in such a way that its effective coupling strength is enhanced at the nuclear surface (see Eq. 1 of Ref. 18]). The overall strength of both functionals is fixed to reproduce $\Delta_{L C S}^{n}$ predicted in ${ }^{120} \mathrm{Sn}$ by the non-empirical functional.

Fig. 2 adds to Fig. 1 the gaps $\left(\Delta_{L C S}^{q}\right)$ calculated from both empirical pairing functionals. Let us first analyze the predicted neutrons gaps displayed in the upper panel of Fig. 2. Keeping in mind that all neutron gaps are the same in ${ }^{120} \mathrm{Sn}$ by construction, one observes, first, that the gaps from REG-V are very close to those obtained with the non-empirical functional, i.e. the isotopic trend is identical whereas the decrease with mass is only slightly less pronounced with REG-V. Moreover the isovector character of REG-S is quite pronounced and opposite to the non-empirical functional. This can be linked to the (surface-peaked) dependence of REG-S on the isoscalar density $\rho_{0}(\mathbf{r})=\rho_{n}(\mathbf{r})+\rho_{p}(\mathbf{r})$ 38. As opposed to a REG-S functional which would only depend on $\rho_{n}(\mathbf{r})$, the dependence on $\rho_{0}(\mathbf{r})$ provides neutron gaps with a sharp increase as a function of $N-Z$ [38. Although one cannot rule out that such an isovector dependence arises when effects beyond the direct two-body interaction are included, the relative disagreement between REG-S and known experimental data makes it rather suspicious at this point. 
Let us now turn to proton gaps. One observes from the lower panel of Fig. 2 that proton gaps from REG-V and the non-empirical functional remain rather close, although the former are systematically smaller than the latter. Proton gaps from REG-S are even smaller and decrease significantly with the nuclear mass, while slightly increasing with the proton number. As a matter of fact, proton gaps from REG-S follow experimental data quite closely. This is a non-trivial result considering, on the one hand, our conjecture that the qualitative difference between experimental neutron and proton gaps is primarily due to Coulomb, and on the other hand that the REG-S pairing functional is isospin invariant.

The qualitatively different behaviors of neutron and proton gaps as a function of isospin asymmetry and nuclear mass can be traced back to the surface-peaked dependence of REG-S on the isoscalar density. In any nucleus, the neutron and proton pairing fields obtained from REG-S are localized in the same region relative to the surface because of the common density dependence on $\rho_{0}(\mathbf{r})$. However, in neutron-rich and/or massive nuclei, where a neutron skin develops, the difference of radii of neutron and proton density distributions makes the surfacepeaked pairing field to overlap more with neutron orbitals than with proton orbitals. This explains the strong increase of neutron gaps with neutron richness and reduction of proton gaps with nuclear mass compared to those obtained from REG-V. Looking more carefully, one notices that the isovector dependence of neutron pairing gaps predicted by REG-S is more prominent than for proton gaps. This calls for a more detailed study of geometrical overlaps of densities, wave functions and density-dependent form factors, as well as an investigation of the localizing effect of the Coulomb barrier on proton states [27]. At this point however, we may conjecture that an empirical REG-S functional depending only (mostly) on $\rho_{n}(\mathbf{r})$ could provide a weaker isovector dependence of neutron gaps [38] on the one hand and mimic Coulomb effects on proton gaps on the other hand 6 . Such a conjecture needs to be tested thoroughly.

\section{Conclusions and outlook}

The first systematic finite-nucleus calculations performed using the Single-Reference Energy Density Functional method and a non-empirical pairing functional derived from low-momentum interactions are reported in the present work. As a first step, the effects of Coulomb and the three-body force on pairing are omitted while only the direct term of the bare two-nucleon interaction is considered. Higher-order effects associated with the coupling to spin, isospin and density fluctuations is postponed to later. The first step taking here towards the construction of non-empirical energy density functionals already constitutes a challenge considering the difficulty to treat the full finite-range and non-locality of the bare nuclear interaction in systematic Energy Density Functional calculations. To do so, the ${ }^{1} S_{0}$ channel of $V_{\text {low } \mathrm{k}}\left(\Lambda \approx 2 f m^{-1}\right)$ is mapped onto a convenient operator form, i.e. a (precise enough) rank-1 separable representation.

For the first time, pairing correlations generated in finite nuclei by the (lowest-order term of the) bare two-nucleon interaction is characterized in a systematic manner. Restricting ourselves to one observable in the present contribution, theoretical and experimental gaps are compared along several isotopic and isotonic chains of semi-magic nuclei. The closeness of theoretical and experimental neutron gaps across several mass regions is striking. Indeed, the non-empirical functional considered here is not final since it is only derived at lowest order in the twobody interaction. On the other hand, theoretical proton gaps are systematically larger than experimental ones which can be attributed to the omission of treating Coulomb in the pairing channel. It is one of the goals of a forthcoming publication to prove this conjecture to be correct [27.

Eventually, predictions from the non-empirical functional are compared to those obtained from empirical local functionals derived from density-dependent zero-range interactions. The characteristics of the latter are analyzed in view of that comparison and a specific modification of their isovector density dependence is suggested to accommodate Coulomb effects and the isovector trend of neutron gaps at the same time.

${ }^{6}$ It would be at the price of dealing with an isospin-symmetry breaking pairing functional. 
Beyond the results briefly summarized in the present contribution, it is the aim of a forthcoming publication to (i) deepen and systematize the analysis, including a more advanced and quantitative evaluation of theoretical pairing gaps (ii) discuss several other observables as well as the effect of Coulomb on proton pairing (iii) use a higher-rank high-precision repre-

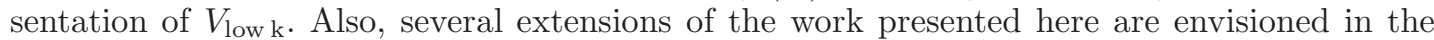
mid-term future. Beyond including the effect of Coulomb on pairing in a way that is numerically tractable, our goal is to study the dependence of the results on the "resolution scale" ( $\Lambda$ cut-off in renormalization group terms) at which the calculations are performed, approximate the nonempirical functional through enriched quasi-local functionals, then use such approximations in codes working in a three-dimensional representation as well as Multi-Reference Energy Density Functional calculations (in the sense of the Generator Coordinate Method). Finally we could employ the latter formalism to study the coupling between density, spin and isospin fluctuations and superfluidity. Ultimately, the effect of three-body forces on pairing in finite nuclei should be investigated, yielding a consistent ab-initio picture.

\section{References}

1. E. Epelbaum, Prog. Part. Nucl. Phys. 57 (2006) 654

2. S. K. Bogner, T. T. S. Kuo, A. Schwenk, Phys. Rept. 386 (2003) 1

3. M. Bender, P.-H. Heenen, P.-G. Reinhard, Rev. Mod. Phys. 75 (2003) 121

4. B. Jancovici, D. H. Schiff, Nucl. Phys. 58 (1964) 678

5. M. Bender, contribution to this volume

6. J. Dobaczewski, W. Nazarewicz, P. G. Reinhard, M. V. Stoitsov, unpublished, arXiv:0708.0441

7. D. Lacroix, T. Duguet, M. Bender, unpublished

8. D. Vautherin, D. M. Brink, Phys. Rev. C5 (1972) 626

9. J. Dechargé, D. Gogny, Phys. Rev. C21 (1980) 1568

10. T. Duguet, M. Bender, P. Bonche, P.-H. Heenen, Phys. Lett. B559 (2003) 201

11. J. Meyer, P. Bonche, J. Dobaczewski, H. Flocard, P. H. Heenen, Nucl. Phys. A533 (1991) 307

12. M. Bender, T. Duguet, Int. J. Mod. Phys. E16 (2007) 222

13. A. Volya, B. A. Brown, V. Zelevinsky, Phys. Lett. B509 (2001) 37

14. N. Pillet, P. Quentin, J. Libert, Nucl. Phys. A697 (2002) 141

15. G. G. Dussel, S. Pittel, J. Dukelsky, P. Sarriguren, Phys. Rev. C76 (2007) 011302(R)

16. S. Hilaire, J.-F. Berger, M. Girod, W. Satula, P. Schuck, Phys. Lett. B531 (2002) 61

17. M. Bender, P. Bonche, T. Duguet, P.-H. Heenen, Nucl. Phys. A723 (2003) 354

18. T. Duguet, K. Bennaceur, P. Bonche, YITP Report Series 112 (2005) B20

19. T. Duguet, K. Bennaceur, T. Lesinski, J. Meyer, INT proceedings 15 (2006) 21

20. S. K. Bogner, A. Schwenk, R. J. Furnstahl, A. Nogga, Nucl. Phys. A763 (2005) 59

21. F. Coester, S. Cohen, B. Day, C. M. Vincent, Phys. Rev. C1 (1970) 769

22. L. P. Gorkov, Sov. Phys. JETP 34 (1958) 505

23. E. M. Henley and L. Wilets, Phys. Rev. 133 (1964) B1118

24. L. G. Cao, U. Lombardo, P. Schuck, Phys. Rev. C74 (2006) 064301

25. F. Barranco, R. A. Broglia, G. Colo', E. Vigezzi, P. F. Bortignon, Eur. Phys. J. A21 (2004) 57

26. D. J. Dean, M. Hjorth-Jensen, Rev. Mod. Phys. 75 (2003) 607

27. T. Lesinski, T. Duguet, K. Bennaceur, unpublished

28. G. E. Brown, A. D. Jackson, The Nucleon-Nucleon Interaction, North-Holland, Amsterdam, 1976

29. T. Duguet, Phys. Rev. C69 (2004) 054317

30. T. Lesinski, unpublished

31. E. Chabanat, P. Bonche, P. Haensel, J. Meyer, R. Schaeffer, Nucl. Phys. A635 (1998) 231

32. T. Duguet and P. Bonche and P.-H. Heenen, J. Meyer, Phys. Rev. C65 (2002) 014311

33. V. Rotival, K. Bennaceur, T. Duguet, unpublished, arXiv:0711.1275

34. S. Hilaire, M. Girod, Eur. Phys. J. A33 (2007) 237

35. M. Anguiano, J. L. Egido, L. M. Robledo, Nucl. Phys. A683 (2001) 227

36. R. B. Wiringa, V. G. J. Stoks, R. Schiavilla, Phys. Rev. C51 (1995) 38

37. J. Dobaczewski, H. Flocard, J. Treiner, Nucl. Phys. A422 (1984) 103

38. T. Duguet, P. Bonche, AIP Conf. Proc. 764 (2005) 277

39. K. Hebeler, T. Lesinski, T. Duguet, A. Schwenk, S. K. Bogner, K. Bennaceur, unpublished 